\title{
Intermediate Logics and the de Jongh property
}

\author{
Dick de Jongh • Rineke Verbrugge • Albert Visser
}

Received: 9 April 2010 / Revised: 14 July 2010 / Accepted: 13 September 2010 /

Published online: 9 October 2010

(C) The Author(s) 2010. This article is published with open access at Springerlink.com

Abstract We prove that all extensions of Heyting Arithmetic with a logic that has the finite frame property possess the de Jongh property.

Keywords Intuitionistic logic $\cdot$ Heyting's arithmetic

Mathematics Subject Classification (2000) $\quad 03 \mathrm{~F} 25 \cdot 03 \mathrm{~F} 30$.

03-02 $\cdot 03 \mathrm{~B} 20 \cdot 03 \mathrm{~F} 50 \cdot 03 \mathrm{~F} 40$

\section{Introduction}

In this paper we generalize de Jongh's theorem that the propositional logic of Heyting's Arithmetic, HA, is precisely Intuitionistic Propositional Logic, IPC. We show that, if we extend $\mathrm{HA}$ with a propositional $\operatorname{logic} \Lambda$, that has the finite frame property, then the resulting theory $\mathrm{HA}(\Lambda)$ has precisely the propositional logic $\Lambda$.

Dedicated to Petr Hájek, on the occasion of his 70th Birthday.

D. de Jongh

ILLC, University of Amsterdam, Room C3.130 B, Building C, Science Park 904, 1098 XH Amsterdam, The Netherlands

e-mail: d.h.j.dejongh@uva.nl

R. Verbrugge

Artificial Intelligence, University of Groningen, PO Box 407, 9700 AK Groningen, The Netherlands e-mail: rineke@ai.rug.nl

A. Visser $(\bowtie)$

Department of Philosophy, Utrecht University, Heidelberglaan 8, 3584 CS Utrecht, The Netherlands e-mail: Albert.Visser@phil.uu.nl 
We explain our result in a bit more detail. Consider a theory $T$ in constructive predicate logic. A propositional formula $\varphi$ is $T$-valid iff, for all substitutions $\sigma$ of formulas of the language of $T$ for propositional variables, we have $T \vdash \sigma(\varphi)$. The set of $T$-valid formulas is the propositional logic of $T$. We will call this logic $\Lambda_{T}$. The de Jongh property for $T$ is the statement that the propositional logic $\Lambda_{T}$ of $T$ is precisely Intuitionistic Propositional Logic, in other words, $\Lambda_{T}=$ IPC.

The original theorem of de Jongh was that Heyting's Arithmetic HA has the de Jongh property. In the past many generalizations have been proved; we will give an enumeration below. In these generalizations the objective was mostly to prove (or in rare cases disprove) the de Jongh property for extensions of HA with some properties like e.g. Church's Thesis. In this paper we will go in a different direction. The idea is to strengthen the logic from the intuitionistic logic to an intermediate one. Intermediate logics are all those logics of strength between intuitionistic and classical logics. Define the de Jongh property for $T$ with respect to an intermediate logic $\Lambda$ as the statement that $\Lambda_{T}=\Lambda$. Our conjecture is the following.

Conjecture 1.1 Let $\mathrm{HA}(\Lambda)$ be the result of extending $\mathrm{HA}$ with $\Lambda$ for all formulas. Then $\Lambda_{\mathrm{HA}(\Lambda)}=\Lambda$.

In this paper we will prove this conjecture for logics $\Lambda$ with the Finite Frame Property, namely,

there exists a class of finite frames $\mathfrak{F}$ such that $\Lambda$ is precisely the logic valid on all models on frames in $\mathfrak{F}$. For this class $\mathfrak{F}$ we then have:

$$
\Lambda=\{\varphi \mid \mathfrak{F}=\varphi\}=\{\varphi \mid \text { for all } \mathcal{M} \text { on } \mathfrak{F}, \mathcal{M} \models \varphi\}
$$

For intermediate logics, as for normal modal logics, the finite frame property $\Lambda$ is in fact equivalent to the ostensibly weaker finite model property (FMP), which expresses that there is a class of finite models $\mathfrak{M}$ such that $\Lambda$ is precisely the logic valid on all models in $\mathfrak{M}$. (See Sect. 3 for more background.)

For logics $\Lambda$ with the finite frame property, indeed $\mathrm{HA}(\Lambda)$ has the de Jongh property with respect to $\Lambda$. As we will discuss in the conclusion (Sect. 6), there seems to be little chance of generalizing the methods of this paper to a more extensive class of intermediate logics.

In our proof we will only employ substitutions of $\Pi_{2}^{0}$-sentences. From this it follows by quite general reasoning, that, assuming that our class of frames is recursive, we have a uniform version of the de Jongh property. This means that, for $\Lambda$ with the finite frame property, there is a single substitution $\sigma^{\star}$ such that $\mathrm{HA}(\Lambda) \vdash \sigma^{\star}(\varphi)$ iff $\Lambda \vdash \varphi$. Or, in a different formulation, there is an embedding of the Lindenbaum Heyting algebra of $\Lambda$ into the Lindenbaum Heyting algebra of $\operatorname{HA}(\Lambda)$.

\section{A brief history of de Jongh's theorem}

The following brief overview of the history of de Jongh's Theorem for propositional logic is adapted from [35]. 
1969 Dick de Jongh proves in an unpublished paper his original theorem that $\Lambda_{\mathrm{HA}}=$ IPC. He uses substitutions of formulas of a complicated form, namely $\forall x(\alpha(x) \vee \neg \alpha(x))$ with $\alpha$ almost negative. As a reminder, a formula is almost negative if it does not contain $\vee$, and $\exists$ only in front of an equation between terms (see [27]). In fact he proves a much stronger result, namely that the logic of relative interpretations in HA is Intuitionistic Predicate Logic. See the extended abstract [7]. De Jongh's argument uses an ingenious combination of Kripke models and realizability.

1973 Harvey Friedman in his paper [9] gives another proof of de Jongh's theorem for $\mathrm{HA}$. He provides a single substitution $\sigma$ mapping the propositional variables to $\Pi_{2}^{0}$-sentences such that HA $\vdash \sigma(\varphi) \Leftrightarrow \mathrm{IPC} \vdash \varphi$. Thus, Friedman shows that IPC is uniformly complete for $\Pi_{2}^{0}$-substitutions in HA. Friedman employs slash-theoretic methods as introduced by Kleene [14].

1973 Craig Smoryński strengthens and extends de Jongh's work in a number of respects in his very readable paper [25]. To state his results we need a few definitions. We write $\mathrm{D}\left(\Pi_{1}\right)$ for the set of disjunctions of $\Pi_{1}^{0}$-sentences, $\operatorname{Prop}\left(\Sigma_{1}\right)$ for propositional combinations of $\Sigma_{1}^{0}$-sentences. Let us remind the reader of some relevant principles (see [27] for extensive discussions).

MP is Markov's Principle MP:

$$
\forall x(A \vee \neg A) \wedge \neg \neg \exists x A \rightarrow \exists x A
$$

$\mathrm{RFN}_{\mathrm{HA}}$ is the formalized uniform reflection principle for $\mathrm{HA}$, where $\forall y A y$ is closed:

$$
\operatorname{Proof}_{\mathrm{HA}}(x,\ulcorner A \bar{y}\urcorner) \rightarrow A y
$$

$\mathrm{TI}(\prec)$ is the transfinite induction scheme for a primitive recursive well-ordering $\prec$ :

$$
\forall x((\forall y \prec x) A y \rightarrow A x) \rightarrow \forall y A y
$$

We have de Jongh's Theorem for the following theories $T$ :

$\mathrm{HA}, \mathrm{HA}+\mathrm{RFN}(\mathrm{HA}), \mathrm{HA}+\mathrm{TI}(\prec)$, and $\mathrm{HA}+\mathrm{MP}$.

For the first three theories we can take the range of our substitutions either $\Sigma_{1}^{0}$ or $\mathrm{D}\left(\Pi_{1}\right)$. For $\mathrm{HA}+\mathrm{MP}$ we can take the range of our substitutions $\operatorname{Prop}\left(\Sigma_{1}\right)$. Smoryński uses Kripke models in combination with the Gödel-RosserMostowski-Kripke-Myhill theorem to prove his results.

1975 Daniel Leivant in his PhD Thesis [16] shows that the predicate logic of interpretations of predicate logic in HA is precisely intuitionistic predicate logic. Leivant's method is proof-theoretical. In fact Leivant shows that one can use as interpretation a fixed sequence of $\Pi_{2}^{0}$-predicates. Leivant's results yield another proof of Friedman's results described above.

1976 de Jongh and Smoryński in their paper [8] show de Jongh's Theorem for HAS. They also show uniform completeness for HAS with respect to a substitution with range among the $\Pi_{2}^{0}$-sentences. 
1981 Yu.V. Gavrilenko in [11] proves de Jongh's Theorem for HA + ECT 0 , i.e., the theory of provable realizability over HA. The principle $\mathrm{ECT}_{0}$ is Extended Church's Thesis (see [27]):

$$
\forall x(A \rightarrow \exists y B y) \rightarrow \exists u \forall x(A \rightarrow \exists v(T u x v \wedge B(U v)))
$$

where $A$ is almost negative and $u$ does not occur free in $A$ and $B$ and $v$ not in $B$. In the formula $\mathrm{ECT}_{0}$ above, $T$ stands for Kleene's $T$-predicate and $U$ for the corresponding result-extracting function [13]. Gavrilenko proves this result as a corollary of the similar result by Smoryński for HA.

1981 As a reminder, the principle DNS stands for 'double negation shift' (see [27]):

$$
\forall x \neg \neg A x \rightarrow \neg \neg \forall x A x
$$

Albert Visser in his Ph.D. thesis [31] provides an alternative proof of de Jongh's theorem for $\mathrm{HA}, \mathrm{HA}+\mathrm{DNS}$, and $\mathrm{HA}+\mathrm{ECT}_{0}$ for $\Sigma_{1}^{0}$-substitutions adapting the method of Solovay's proof of the arithmetical completeness of Löb's logic for substitutions in PA [24]. In fact, his proof extends to the same theories extended with appropriate reflection principles or transfinite induction over primitive recursive well-orderings.

1985 In his [33], Albert Visser provides an alternative proof of de Jongh's uniform completeness theorem employing a single $\Sigma_{1}^{0}$-substitution. The proof is verifiable in $\mathrm{HA}+\mathrm{con}(\mathrm{HA})$. Here, con( $\mathrm{HA})$ formalizes the consistency of Heyting Arithmetic. Note that de Jongh's theorem implies con(HA), so the result is, in a sense, optimal. Visser's proof uses the NNIL-algorithm, an algorithm that is used to characterize the admissible rules for $\Sigma_{1}^{0}$-substitutions. See also [36].

1991 Jaap van Oosten in his paper [30] provides a more perspicuous version of de Jongh's semantical proof of de Jongh's theorem for (non-relativized) interpretations of predicate logic. Van Oosten uses Beth models and realizability. See also [29].

1996 Using the methods developed by Visser in [32] and by de Jongh and Visser in [6], one can prove uniform completeness with respect to $\Sigma_{1}^{0}$-substitutions for $\mathrm{HA}+\mathrm{ECT}_{0}, \mathrm{HA}+\mathrm{ECT}_{0}+\mathrm{RFN}\left(\mathrm{HA}+\mathrm{ECT}_{0}\right)$, and $\mathrm{HA}+\mathrm{TI}(\prec)+\mathrm{ECT}_{0}$.

It is well known that the de Jongh property does not hold for HA $+\mathrm{MP}+\mathrm{ECT}_{0}$. Consider the formulas $\chi$ and $\rho$, which are defined as follows.

- $\chi:=(\neg p \vee \neg q)$,

- $\rho:=[(\neg \neg \chi \rightarrow \chi) \rightarrow(\chi \vee \neg \chi)] \rightarrow(\neg \neg \chi \vee \neg \chi)$

Clearly, $\rho$ is not provable in IPC. We use $\mathbf{r}$ for Kleene realizability. In his classical paper [23], G.F. Rose showed that: $\exists e \forall \sigma \in \operatorname{sub}_{\mathrm{HA}} \mathbb{N} \models e \mathbf{r} \sigma(\rho)$. Here sub $\mathrm{HA}_{\mathrm{HA}}$ is the set of substitutions from the propositional variables to sentences of the arithmetical language. Thus, Rose refuted a conjecture of Kleene that a propositional formula is IPC-provable if all its arithmetical instances are (truly and classically) realizable. Note 
the remarkable fact that one and the same realizer realizes all instances! Inspecting the proof, one sees that only a small part of classical logic is involved in the verification of realizability: Markov's Principle. See David McCarthy's paper [19] for a detailed analysis. Thus we obtain:

$$
\exists e \forall \sigma \in \mathrm{Sub}_{\mathrm{HA}} \mathrm{HA}+\mathrm{MP} \vdash e \mathbf{r} \sigma(\rho) .
$$

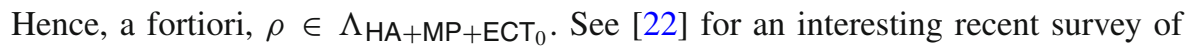
propositional realizability logic.

\section{Intermediate logics and the finite model property}

Intermediate logics (also called superintuitionistic logics) are the logics between IPC and CPC, classical propositional logic, i.e. the sets of of formulas closed under IPCdeduction and uniform substitution. Most anything one needs to know about these logics can be found in [4]. We will enumerate the best known of these logics and mention a few basic facts, mainly concerning the finite model property. The well-known theorem for normal modal logics that the finite frame property and the finite model property for a modal logic $L$ coincide (see e.g. [1]), applies to intermediate logics as well [4]. This means that every sentence not provable from the logic cannot only be refuted in a finite model of the logic, it can also be refuted in a frame validating the logic. So, it is appropriate to use the terminology that a logic has the finite model property (FMP) if there is a class of finite frames for which it is complete. As in the case of modal logics, not all intermediate logics are complete with respect to a class of frames, and not all those which are complete for such a class have the FMP. But all except one of the well-known intermediate logics which we will now discuss are known to be complete with respect to a class of finite frames.

LC, Dummett's logic, axiomatized by, e.g. $(\varphi \rightarrow \psi) \vee(\psi \rightarrow \varphi)$, is complete with respect to the finite linear frames.

KC, Jankov's logic (also called the logic of weak excluded middle, whereas real intuitionists might prefer, if anything, the logic of testability), axiomatized by $\neg \varphi \vee \neg \neg \varphi$, is complete with respect to the finite frames with a unique endpoint.

KP, the logic of Kreisel and Putnam, axiomatized by

$$
(\neg \varphi \rightarrow \psi \vee \chi) \rightarrow(\neg \varphi \rightarrow \psi) \vee(\neg \varphi \rightarrow \chi)
$$

It is complete with respect to the finite partial orderings satisfying the property: For each $u$ and each set $X$ of points succeeding $u$, there exists a $v$ accessible from $u$ such that all points of $X$ are accessible from $v$ and every endpoint above $v$ is also above some point from $X$. KP was the first logic shown to have the disjunction property: If $\vdash \varphi \vee \psi$, then $\vdash \varphi$ or $\vdash \psi$, where $\vdash$ stands for provability in the given logic (here $\mathrm{KP})[15]$. 
$T_{n}$, the Gabbay-deJongh logics, which are complete with respect to the finite trees which have splittings of exactly $n$, i.e. each node has exactly $n$ immediate successors. $\mathrm{T}_{\mathrm{n}}$ is axiomatized by

$$
\bigwedge_{k \leq n+1}\left(\left(\varphi_{k} \rightarrow \bigvee_{j \neq k} \varphi_{j}\right) \rightarrow \bigvee_{j \neq k} \varphi_{j}\right) \rightarrow \bigvee_{k \leq n+1} \varphi_{k}
$$

$\mathrm{T}_{1}$ coincides with LC. $\mathrm{T}_{\mathrm{n}}$-frames for $n>1$ do not have a first order definition. Moreover, $T_{\mathrm{n}}$ for $n>1$ is not canonical ([4]). However, it is first-order definable on finite frames: it is characterized by the condition that every point has at most $n$ immediate successors. Finally, $\mathrm{T}_{\mathrm{n}}$ for $n>1$ has the disjunction property [5].

$\mathrm{BD}_{n}$, the depth $n$ logics are complete with respect to the finite partial orderings (but also to the trees, or to the splitting trees) of depth $n$. $\mathrm{BD}_{1}$ is classical logic, and is axiomatized by Peirce's Law, $((\varphi \rightarrow \psi) \rightarrow \varphi) \rightarrow \varphi$. An axiomatization of $\mathrm{BD}_{\mathrm{n}}$ for $n>1$ is obtained by iteratedly substituting Peirce's Law into itself, e.g. $\mathrm{BD}_{2}$ is axiomatized by $((\varphi \rightarrow(((\psi \rightarrow \chi) \rightarrow \psi) \rightarrow \psi)) \rightarrow \varphi) \rightarrow \varphi$.

Sc, Scott's logic, axiomatized by $((\neg \neg \varphi \rightarrow \varphi) \rightarrow(\varphi \vee \neg \varphi)) \rightarrow \neg \varphi \vee \neg \neg \varphi$. It is complete with respect to the finite partial orderings satisfying the property: In each generated subframe all the endpoints are connected by an $R, R^{-1}$-chain containing only points of depth 0 or 1 (or equivalently the finite frames which do not have a p-morphism onto the asymmetric four-element tree of depth 3). This is not equivalent to a first-order definition. Like $\mathrm{T}_{n}$ and KP, Scott's logic also has the disjunction property $[4,15]$.

ML, Medvedev's logic of finite problems [20] has the finite frame property (it is complete with respect to the finite boolean algebras without their top element), but it has no known axiomatization and is not known to be decidable. It contains Scott's logic and KP and is contained in Jankov's logic. It is not finitely axiomatizable and its infinite axiom systems will need an infinity of atoms [18]. ML coincides with the set of formulas all whose essentially negative substitution instances are provable in $\mathrm{KP}$, or stated in another form, it is the logic of the valid schemata obtained by adding $\neg \neg p \rightarrow p$ for atoms only to KP (due to [17], see also [3]).

The Propositional Logic of Realizability. Plisko [22] discusses several variants out of which we choose the logic of the effectively realizable formulas, although for most purposes it makes little difference. The logic has no known axiomatization, it is not known whether it has the finite model property or is decidable. It does have the disjunction property. From our earlier remarks it follows that it contains Scott's logic with $\neg \psi \vee \neg \theta$ substituted for the sole variable in Scott's axiom. Just like ML, the Propositional Logic of Realizability is contained in KC, but it has been shown to neither contain nor be contained in ML.

All logics using only $\rightarrow, \wedge, \neg$ in their axioms have the finite model property, but none but IPC itself have the disjunction property (see [4]). The same is true for logics with axioms using only NNIL-formulas (No Nesting of Implications on the Left); in fact, these logics are the same as the ones using only $\rightarrow$ (see [38]). 


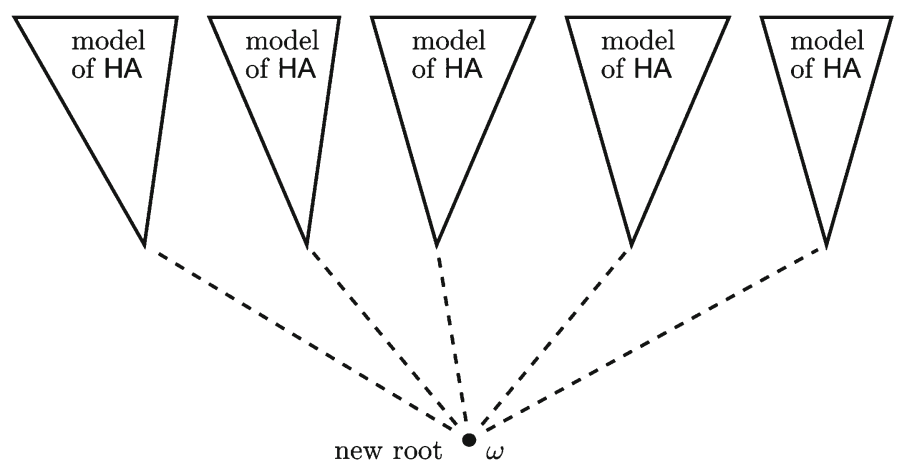

Fig. 1 Scheme of lemma 4.1: A new model of HA is constructed by equipping a set of models of HA with a new root, and attaching the standard model $\omega$ to it

\section{Arithmetics and finite frames}

In this section, we prove our main result. We follow Smoryński's classical paper [25]. In that paper Smoryński gives two proofs. We will use the second more complicated one, which is more flexible for applications. But first we will sketch in a few lines the first proof which gives us a partial result, and indicate why it does not generalize. The idea (of both proofs) is, given a propositional Kripke-model, to construct a Kripke model for $\mathrm{HA}$ on the same frame, and then to exhibit arithmetical sentences with exactly the same forcing behavior as the propositional variables on the propositional model.

First, one should realize that any node $k$ in a model $\mathcal{K}$ is attached to a classical structure $\mathcal{M}_{k}$ (not necessarily a model of HA [2]). The simple proof now starts with the basic lemma:

Lemma 4.1 Given a (non-rooted) model of $\mathrm{HA}$ (or equivalently a set of models for $\mathrm{HA}$ ), one can obtain a new model of $\mathrm{HA}$ by equipping this non-rooted model with a root to which the standard model of the natural numbers is attached. See Fig. 1.

Proof Assume $\mathcal{K}, \mathcal{K}^{\prime}, r$ as in the statement of the lemma. Assume also that $r \Vdash A(0) \wedge$ $\forall x(A(x) \rightarrow A(x+1))$. It is sufficient to show that $r \Vdash \forall x A(x)$, since the $\mathcal{K}$-part of the model already satisfies (internal) induction. We have that $r \Vdash A(0) \wedge(A(n) \rightarrow$ $A(n+1))$ for each natural number $n$. This enables us, by applying external induction, to conclude that the new root forces $A(n)$ for each $n$, from which $r \Vdash \forall x A(x)$ follows.

The simple proof now continues using the fact that IPC is complete with respect to the finite trees that are everywhere splitting (each node has at least two immediate successors). One builds a propositional Kripke-model on the same tree by starting with non-standard models for PA (and thus for HA!) on the endpoints, and attaching the standard model to each of the other nodes. By the basic lemma 4.1, using induction on the depth of the nodes, this is a model of HA.

The non-standard models at the endpoints are chosen to be incomparable in the following sense: each of them has its own $\Sigma_{1}^{0}$-sentence, which, in PA, contradicts 
all the others. Then, because one has a finite everywhere splitting tree, each node is completely determined by the endpoints which are accessible from it. This is sufficient to create for each node a sentence true on exactly that node (and its successors). Finally, disjunctions of those sentences will characterize arbitrary upward closed subsets of the model, in particular those determined by the valuations of the propositional variables in the propositional model.

To those intermediate logics that are complete with respect to a set of finite splitting trees, this method will immediately apply. The point is that we do not only get a model of $\mathrm{HA}$ but, because of the form of the frame, also of $\mathrm{HA}(\Lambda)$. This is immediately clear for $\varphi\left(\alpha_{1}, \ldots, \alpha_{n}\right)$ if $\varphi\left(p_{1}, \ldots, p_{n}\right)$ is a member of $\Lambda$ and $\alpha_{1}, \ldots, \alpha_{n}$ are arithmetic sentences with constants for the elements of the appropriate domains of the models. If in such a case $\alpha_{1}, \ldots \alpha_{n}$ have free variables, then the truth value of the universally quantified version of $\varphi\left(\alpha_{1}, \ldots, \alpha_{n}\right)$ depends only on the value of sentences arising by substitution in $\varphi\left(p_{1}, \ldots, p_{n}\right)$, and, since we declared these to be true already, the universally quantified forms have to be true as well. In particular, one obtains in this manner the de Jongh property for $\mathrm{HA}$ with respect to the logics $\mathrm{T}_{\mathrm{n}}$ (for $n>1$ ) and $\mathrm{BD}_{\mathrm{n}}$, but not for $\mathrm{KC}, \mathrm{KP}$, and $\mathrm{ML}$. Also not for $\mathrm{T}_{1}=\mathrm{LC}$, because linear frames do not split everywhere.

Especially, the need to characterize each node exactly by the endpoints accessible from it seems essential in this setup of the proof. So, for the general case we will need a different way to proceed. One basic idea remains the same. We want to fit a root to a number of models. Smoryński's second method does this in a much more sophisticated manner than the first, and with this method one can under certain strict conditions have a root with a non-standard model attached to it. To set the stage, we need a sequence of lemmas. We start with a few well-known lemmas on Kripke models of HA. For definitions of forcing in such Kripke models, see [25] and [28].

Lemma 4.2 Suppose $\mathcal{K}$ is a Kripke model of $\mathrm{HA}$. Let $A \vec{x}$ be a $\Sigma_{1}^{0}$-formula. Then, for any node $k$ and any $\vec{d}$ in $D_{k}$, we have $k \Vdash A \vec{d}$ iff $\mathcal{M}_{k} \models A \vec{d}$.

Proof We first prove, by induction on the complexity of $A$, that, for any $\Delta_{0}^{0}$-formula $A \vec{x}$, and any node $k$ and any $\vec{d}$ in $D_{k}$, we have $k \Vdash A \vec{d}$ iff $\mathcal{M}_{k} \models A \vec{d}$. The proof uses the fact that HA proves the decidability of $\Delta_{1}^{0}$-formulas. We treat the case of restricted universal quantification.

Suppose we have the desired property for $B y \vec{x}$ in $\Delta_{0}^{0}$. If $k \Vdash \forall y<e B y \vec{d}$, then evidently, for all $d^{\prime}<\mathcal{M}_{k} e, k \Vdash B d^{\prime} \vec{d}$. Hence, by the Induction Hypothesis, for all $d^{\prime}<\mathcal{M}_{k} e, \mathcal{M}_{k} \models B d^{\prime} \vec{d}$. Hence, $\mathcal{M}_{k}=\forall y<e$ By $\vec{d}$.

Conversely, suppose (a) $\mathcal{M}_{k} \models \forall y<e B y \vec{d}$. In order to derive a contradiction, suppose (b) $k \Vdash \exists y<e \neg B y \vec{d}$. It follows by the Induction Hypothesis and writing out the negation and restricted existential quantification, that $\mathcal{M}_{k} \models \exists y<e \neg B y \vec{d}$, contradicting (a). So, canceling (b), we conclude $k \nVdash \exists y<x \neg B y \vec{d}$. Then, by decidability, $k \Vdash \neg \exists y<e \neg B y \vec{d}$. So, by intuitionistic predicate logic, $k \Vdash \forall y<e \neg \neg B y \vec{d}$. By decidability again, $k \Vdash \forall y<e B y \vec{d}$.

The step from $\Delta_{0}^{0}$ to $\Sigma_{1}^{0}$ is easy.

The following lemma is due to Wim Ruitenburg. 
Lemma 4.3 Let $\mathcal{K}$ be a model of $\mathrm{HA}$. Let $A \vec{x}$ be a $\Pi_{2}^{0}$-formula. Then, for any node $k$ and for any $\vec{d}$ in $D_{k}$, we have: $k \Vdash A \vec{d}$ iff, for all $k^{\prime} \geq k, \mathcal{M}_{k^{\prime}} \models A \vec{d}$.

Proof Suppose $A \vec{x}$ is $\forall \vec{y} S \vec{y} \vec{x}$, where $S$ is $\Sigma_{1}^{0}$. We have:

$$
\begin{aligned}
k \Vdash \forall \vec{y} S \vec{y} \vec{d} & \Leftrightarrow \forall k^{\prime} \geq k \forall \vec{e} \in D_{k^{\prime}} k^{\prime} \Vdash S \vec{e} \vec{d} \\
& \Leftrightarrow \forall k^{\prime} \geq k \forall \vec{e} \in D_{k^{\prime}} \mathcal{M}_{k^{\prime}} \models S \vec{e} \vec{d} \\
& \Leftrightarrow \forall k^{\prime} \geq k \mathcal{M}_{k^{\prime}}=\forall \vec{y} S \vec{y} \vec{d}
\end{aligned}
$$

This gives us the desired property.

Next, we need some basic insights from the theory of interpretations.

Lemma 4.4 Let $T$ be any $R E$ theory. Then $\mathrm{Q}+\operatorname{con}(T)$ interprets $T$.

Here Q is Robinson's Arithmetic, a very weak arithmetical theory introduced by Tarski, Mostowski and Robinson in their book [26].

This is a general version of a fundamental theorem due to Hilbert and Bernays, which was worked out by Wang. The proof was simplified by Feferman. The methods that lead to the general version are due to among others Solovay et al. See [37], for context, explanation and references. A very crude explanation of the result is as follows. The Model Existence Lemma tells us that, if a theory $T$ is consistent, there is a model $\mathcal{N}$ of $T$. A moment's reflection shows that the construction of this model, via the Henkin construction, is syntactical in nature. By fine-tuning the argument, we can see that inside a theory that contains the consistency statement of $T$ we can construct an interpretation of $T$. Here the heuristic is that interpretations are something like uniformly defined internal models.

Thus, inside any model $\mathcal{M}$ of $\mathrm{Q}+\operatorname{con}(T)$ we can construct a model $\mathcal{M}^{\prime}$ of $T$. Moreover, if $\mathcal{M}$ is a model of PA, and if $T$ is an arithmetical theory extending $\mathrm{Q}$, then we can easily prove, using an argument that is essentially due to Dedekind, that $\mathcal{M}^{\prime}$ is an end-extension of $\mathcal{M}$ modulo a definable embedding. The idea is that inside $\mathcal{M}$ we can define the obvious function that maps the numbers of the theory into the numbers of the internal model $\mathcal{M}^{\prime}$ by recursion. We can prove by induction that the numbers of $\mathcal{M}$ are mapped to an initial segment of $\mathcal{M}^{\prime}$. We formulate this in a lemma. For precise discussions of this and similar lemmas, see [12].

Lemma 4.5 Suppose $T$ is an arithmetical theory extending $Q$. Then every model $\mathcal{M}$ of PA $+\operatorname{con}(T)$ contains an internally definable end-extension (modulo a definable embedding) satisfying $T$.

Finally, we formulate Smoryński's fundamental lemma. Consider a non-rooted Kripke model $\mathcal{K}$ of $\mathrm{HA}$ and a model $\mathcal{M}$ of PA. Suppose $\mathcal{K}$ is definable in $\mathcal{M}$. This means that the set of nodes $K$ of $\mathcal{K}$ is definable, that the ordering on the nodes is definable, that there is a formula $\delta(k, d)$ giving the domain elements of the node $k$, and that there are arithmetical formulas $A_{P}(k, \vec{d})$ representing $k \Vdash P \vec{d}$. Moreover, we ask that $\mathcal{M}$ verifies basic properties like the fact that the relation between the nodes is a partial ordering. We have: 
Lemma 4.6 There is a definable embedding of $\mathcal{M}$ as an initial segment of the model associated with each node of $\mathcal{K}$. This embedding is unique in the strong sense that there can be only one definable embedding that commutes with 0 and successor. Thus we can form a rooted model $\mathcal{K}^{+}$by adding $\mathcal{M}$ as a new root to $\mathcal{K}$. We have: $\mathcal{K}^{+}$is a model of $\mathrm{HA}$.

The proof of this result is in Smoryński's classical paper [25]. The idea is a simple extension of the idea of the basic lemma 4.1. Given a nonrooted model of HA, we cannot just add an arbitrary non-standard model as the root, since such a model does have induction for its own language but not for the enriched language in which we can also talk about $\mathcal{K}$. However, if $\mathcal{K}$ is internally definable this problem disappears and we can add the non-standard model as a root. One could say that the non-standard model internally thinks it is standard and that as soon as it can talk about $\mathcal{K}$ the earlier external argument can be internalized.

We are now ready for the main construction, which diverges from Smoryński's proof [25].

Theorem 4.1 Suppose that $\Lambda$ is the logic of a given class of finite frames $\mathfrak{F}$. Let $\mathrm{HA}(\Lambda)$ be the result of extending $\mathrm{HA}$ with $\Lambda$ for all formulas. Then

$$
\Lambda_{\mathrm{HA}(\Lambda)}=\Lambda
$$

i.e. the propositional logic of $\mathrm{HA}(\Lambda)$ is $\Lambda$. Our result works both when we consider logics of substitutions of formulas and when we consider the logics of substitutions of sentences.

Proof Let $\Lambda$ be the logic of a given class of finite frames $\mathfrak{F}$. Suppose $\Lambda \nvdash \varphi$. There is a finite model $\mathcal{K}$ with frame in $\mathfrak{F}$, such that $\mathcal{K} \nVdash \varphi$. Let the ordering of our model be $\preceq$. We can arrange it so that the nodes of $\mathcal{K}$ are $0, \ldots, n-1$ and, if $i \preceq j$, then $i \leq j$. We define:

- $\operatorname{incon}^{0}(\mathrm{PA}):=\perp$, incon ${ }^{k+1}(\mathrm{PA}):=\operatorname{prov}_{\mathrm{PA}}\left(\right.$ incon $\left.^{k}(\mathrm{PA})\right)$,

- $C_{k}:=\neg \operatorname{incon}^{k}(\mathrm{PA}) \wedge$ incon $^{k+1}(\mathrm{PA})$,

- $\bar{C}_{0}(x):=$ incon $^{1}(\mathrm{PA})$,

- $\bar{C}_{k+1}(x):=\neg \operatorname{proof}_{\mathrm{PA}}\left(x, \operatorname{incon}^{k}(\mathrm{PA})\right) \wedge \operatorname{incon}^{k+2}(\mathrm{PA})$.

We note that the $C_{i}$ are mutually exclusive. We define $T_{i}$, for $0 \leq i<n$, by $T_{i}:=\mathrm{PA}+C_{n-i-1}$. By Löb's Theorem and $\Sigma_{1}^{0}$-soundness, each of the $T_{i}$ is consistent. Moreover, again by Löb's Theorem, for $i<n-1$, we have $T_{i} \vdash \operatorname{con}\left(T_{i+1}\right)$. Let $\mathcal{N}_{0}$ be any model of $T_{0}$. By Lemma 4.4, we can construct an internal model, say $\mathcal{N}_{1}$ of $T_{1}$, in $\mathcal{N}_{0}$. The model $\mathcal{N}_{1}$ will be an end-extension of $\mathcal{N}_{0}$. We iterate this construction, obtaining an internal model $\mathcal{N}_{i+1}$ of $T_{i+1}$ in $\mathcal{N}_{i}$. Since 'being an internal model of' and 'being an end-extension of' are transitive relations, we find that if $i \leq j \leq n-1$, then $\mathcal{N}_{j}$ is an internally defined end-extension of $\mathcal{N}_{i}$.

We now construct a Kripke model $\mathcal{S}$ by making $\mathcal{N}_{k}$ the model associated to the node $k$ and taking over the ordering of $\mathcal{K}$. By Smoryński's Lemma 4.6, we find that $\mathcal{S}$ is a model of HA. Since the frame of our model is in $\mathfrak{F}$, we see that $\mathcal{S}$ satisfies $\operatorname{HA}(\Lambda)$. 
Consider any proposition $p$ in $\mathcal{K}$. We want to find an arithmetical formula $\sigma(p)$ such that, for all $k<n, \mathcal{S}, k \Vdash \sigma(p)$ iff $\mathcal{K}, k \Vdash p$. A first choice to consider would be $\sigma(p):=\bigvee_{\mathcal{K}, k \Vdash p} C_{n-k-1}$. However, it is easy to see this won't wash. Consider, for example, $i \prec j$ and suppose $\mathcal{S}, i \Vdash \sigma(p)$. Then $\mathcal{S}, i \Vdash C_{m}$, for some $m$ with $\mathcal{K}, m \Vdash p$. It follows that $\mathcal{N}_{i} \models C_{m}$, and hence that $m=n-i-1$. By persistence, we find that $\mathcal{S}, j \Vdash C_{n-i-1}$, but then $\mathcal{N}_{j} \models C_{n-i-1}$. A contradiction.

So this does not work. In the light of Ruitenburg's lemma 4.3, we can diagnose the problem as follows: the sentence $\sigma(p)$ is not constructively equivalent to a $\Pi_{2}^{0}$-sentence or some other kind of sentence that gives us a transfer from classical satisfaction and constructive forcing. However, our $\sigma(p)$ is classically equivalent to a $\Pi_{2}^{0}$-sentence, and therein lies the simple solution. Suppose $\{k \mid \mathcal{K}, k \Vdash p\}=\left\{k_{0}, \ldots, k_{m-1}\right\}$. We define:

- $\sigma^{\star}(p):=\forall x_{0}, \ldots x_{m-1}\left(\bar{C}_{n-k_{0}-1}\left(x_{0}\right) \vee \cdots \vee \bar{C}_{n-k_{(m-1)}-1}\left(x_{m-1}\right)\right)$.

It is easy to see that constructively $\sigma^{\star}(p)$ is equivalent to a $\Pi_{2}^{0}$-sentence and that classically $\sigma^{\star}(p)$ is equivalent to $\sigma(p)$.

By Ruitenburg's Lemma 4.3, we find:

$$
\mathcal{S}, i \Vdash \sigma^{\star}(p) \Leftrightarrow \mathcal{K}, i \Vdash p .
$$

Hence, by induction, we find that, for any $\psi$ :

$$
\mathcal{S}, i \Vdash \sigma^{\star}(\psi) \Leftrightarrow \mathcal{K}, i \Vdash \psi \text {. }
$$

So, $\mathcal{S}, 0 \nVdash \sigma^{\star}(\varphi)$.

Remark 4.2 A remarkable aspect of the above proof is that, where Smoryński used Rosser-style self-reference, it only employs Gödelean self-reference-since it uses Löb's Theorem.

Remark 4.3 We note that we can employ a fixed series of models in our main argument that can be chosen independently of the finite frames. This uses an argument originally due to Harvey Friedman [10]. We define by Carnap's version of the Fixed Point Lemma a formula $A(x)$ such that:

$$
\mathrm{PA} \vdash \forall x\left(A(x) \leftrightarrow\left(\operatorname{con}(\mathrm{PA}+A(x+1)) \vee \exists y \leq x \operatorname{proof}_{\mathrm{PA}}(y, \neg A(0))\right)\right) .
$$

Let $T_{i}:=\mathrm{PA}+A(i)+\operatorname{incon}(\mathrm{PA}+A(i))$. Let $C_{i}:=\operatorname{con}\left(T_{i+1}\right) \wedge \operatorname{incon}\left(T_{i}\right)$. We easily see that the $T_{i}$ are consistent and that $T_{i}$ is equivalent to PA $+C_{i}$. Moreover, the $C_{i}$ are incompatible. We note that, by Löb's Theorem, $T_{i} \vdash \operatorname{con}\left(T_{i+1}\right)$. We now start with a model $\mathcal{N}_{0}$ of $T_{0}$ in which we construct an internally definable end-extension $\mathcal{N}_{1}$ that is a model of $T_{1}$, etc. Thus we obtain a sequence of models $\mathcal{N}_{i}$, where each next model is a definable end-extension of the previous one. Moreover, $\mathcal{N}_{i} \models C_{i}$.

Can we extend the above proof to other arithmetical theories besides $\mathrm{HA}$ as basis? We note that we can extend it to weaker theories like $i-I \Sigma_{1}$, the intuitionistic version of $I \Sigma_{1}$, since the construction of an end-extension from a consistency statement already 
works in the classical theory $I \Sigma_{1}$ [12]. Secondly, the proof also works for stronger theories like HA plus uniform reflection, or HA extended with an RE set of negations that are $\Pi_{2}^{0}$-conservative over PA.

We have one somewhat more interesting extension of our result, for intermediate logics with what we will call the endpoint replacement property:

Definition 4.4 Consider an intermediate logic $\Lambda$.

(1) Let $\mathfrak{F}$ be a class of frames for the logic. We say that $\Lambda$ has the endpoint replacement property with respect to $\mathfrak{F}$ if the following holds. Take any $\mathcal{F}$ in $\mathfrak{F}$, a set of endpoints $\left\{u_{i} \mid i \in I\right\}$ of $\mathcal{F}$, and a set $\left\{\mathcal{M}_{i} \mid i \in I\right\}$ of (possibly infinite) models of $\Lambda$ with roots $\left\{r_{i} \mid i \in I\right\}$. Let $\mathcal{G}$ be the frame that is the result of replacing the endpoints $\left\{u_{i} \mid i \in I\right\}$ by the frames of $\left\{\mathcal{M}_{i} \mid i \in I\right\}$. Then any model $\mathcal{M}$ on $\mathcal{G}$ which is such that its submodels generated by $\left\{r_{i} \mid i \in I\right\}$ are exactly the $\left\{\mathcal{M}_{i} \mid i \in I\right\}$ will validate $\Lambda$.

(2) $\Lambda$ has the endpoint replacement property if $\Lambda$ has the endpoint replacement property with respect to its class of frames.

Of the logics we mentioned, the following have the endpoint replacement property: IPC, KC, LC, and $T_{n}$. The logics KP, Sc, ML, and $B D_{n}$ do not have the property with respect to a class of frames for which they are complete. On the negative side: for KP and Sc this is obvious from the fact that the simple 3-element fork which is a frame for both is no longer a frame for either when attached to an endpoint of itself. On the positive side, we will just show the most complicated case: the $T_{n}$.

Proposition 4.5 $\mathrm{T}_{\mathrm{n}}$ has the endpoint replacement property.

Proof Let $\mathcal{F}$ be a frame for $\mathrm{T}_{\mathrm{n}}$, let $\left\{u_{i} \mid i \in I\right\}$ be a set of endpoints of $\mathcal{F}$, and let $\left\{\mathcal{M}_{i} \mid i \in I\right\}$ be a set of models of $\mathrm{T}_{\mathrm{n}}$ with roots $\left\{r_{i} \mid i \in I\right\}$. Let $\mathcal{M}$ be a model on the frame $\mathcal{G}$ that is the result of replacing the endpoints $\left\{u_{i} \mid i \in I\right\}$ by the frames of $\left\{\mathcal{M}_{i} \mid i \in I\right\}$ such that the models generated by the $r_{i}$ are exactly the $\mathcal{M}_{i}$. We have to show that the assumption that $T_{\mathrm{n}}\left(\varphi_{1}, \ldots, \varphi_{n+1}\right)$ is falsified in $\mathcal{M}$ will lead to a contradiction. We can then assume without loss of generality that the root satisfies the antecedent of $\mathrm{T}_{\mathrm{n}}$ for $\varphi_{1}, \ldots, \varphi_{n+1}$ and falsifies each of $\varphi_{1}, \ldots, \varphi_{n+1}$. Let us define $N$ as the set of nodes in $\mathcal{M}$ that falsify each of $\varphi_{1}, \ldots, \varphi_{n+1}$. This will be a downward closed set containing the root, falsifying $\mathrm{T}_{\mathrm{n}}\left(\varphi_{1}, \ldots, \varphi_{n+1}\right)$ everywhere. Since for each $k \leq n+1, \bigvee_{j \neq k} \varphi_{j}$ is falsified at each $v \in N$, also $\varphi_{k} \rightarrow \bigvee_{j \neq k} \varphi_{j}$ is false at $v$ for some $k \leq n+1$. This means that for each $v \in N$, there is a $k \leq n+1$ and a $w_{k}$ with $v \leq w_{k}$ such that $w_{k}$ makes $\varphi_{k}$ true but no $\varphi_{j}$ for $j \neq k$. Note that such nodes $w_{k}$ and $w_{j}$ for different $k$ and $j$ are always incomparable. Note also that $x \Vdash \mathrm{T}_{\mathrm{n}}$ iff $x \in N$.

Let us first consider the case that one of the $r_{i}$ is in $N$. That is impossible, since then $r_{i} \Vdash \mathrm{T}_{\mathrm{n}}\left(\varphi_{1}, \ldots, \varphi_{n+1}\right)$ whereas the model $\mathcal{M}_{i}$ is supposed to be a model for $\mathrm{T}_{\mathrm{n}}$. The second possibility is that none of the $r_{i}$ are in $N$. Note that then, if $\mathcal{M}_{i}$ contains any $w_{k}$-node as described above, its root $r_{i}$ will have to be a $w_{k}$-node, and there cannot be $w_{j}$-nodes for any $j \neq k$ in $\mathcal{M}_{i}$. If we now restore the endpoints $\left\{u_{i} \mid i \in I\right\}$ in $\mathcal{M}$ in place of the models $\left\{\mathcal{M}_{i} \mid i \in I\right\}$ and define

$$
V^{\prime}\left(p_{m}\right)=\left\{w \in \mathcal{F} \backslash\left\{u_{i} \mid i \in I\right\} \mid w \Vdash \varphi_{m}\right\} \cup\left\{u_{i} \mid r_{i} \Vdash \varphi_{m}\right\},
$$


then it is obvious that for this new valuation, for each $v \in N$ (and in particular for the root of $\mathcal{F}), v \mid \nvdash^{\prime} \mathrm{T}_{\mathrm{n}}\left(p_{1}, \ldots, p_{n+1}\right)$, since one of the $p_{k} \rightarrow \bigvee_{j \neq k} p_{j}$ will be falsified at the appropriate $w_{k}$. Thus, the resulting model will falsify $\mathrm{T}_{\mathrm{n}}\left(p_{1}, \ldots, p_{n+1}\right)$. This is again a contradiction, because $\mathcal{F}$ is a frame for $T_{n}$.

Theorem 4.6 Suppose that $\Lambda$ has both the finite model property and the endpoint replacement property with respect to a class $\mathfrak{F}$ of finite frames. Suppose that $U$ is a consistent extension of $\mathrm{HA}(\Lambda)$ with an RE set of negations $N$. Then $U$ has the de Jongh property for $\Lambda$.

Before we turn to the proof of our theorem, we remind the reader of some basic facts concerning Rosser sentences. Let $V$ be any consistent RE extension of $i$-EA, the intuitionistic version of Elementary Arithmetic. Using Craig's trick, we can arrange that the axiom set of $V$ is given by an elementary predicate $\alpha$. This predicate can be taken to be a $\Delta_{1}^{0}(i$-EA)-formula. This means that both $\alpha x$ and $\neg \alpha x$ are $i$-EA-provably equivalent to a $\Sigma_{1}^{0}$-formula and that $\alpha x$ is $i$-EA-provably decidable. Using this predicate, we can find a reasonable arithmetization proof $_{V}$, the proof-predicate for $V$, such that $\operatorname{proof}_{V}(x, y)$ is $\Delta_{1}^{0}\left(i\right.$-EA). Let $\operatorname{prov}_{V}(x)$ be $\exists y \operatorname{proof}_{V}(y, x)$.

Consider formulas $A$ and $B$. Suppose $A=\exists x A_{0} x$ and $B=\exists y B_{0} y$. We define:

- $A \leq B: \leftrightarrow \exists x\left(A_{0} x \wedge \forall y<x \neg B_{0} y\right)$,

- $A<B: \leftrightarrow \exists x\left(A_{0} x \wedge \forall y \leq x \neg B_{0} y\right)$,

- $(A<B)^{\perp}: \leftrightarrow B \leq A$,

- $(A \leq B)^{\perp}: \leftrightarrow B<A$.

Using Gödel's Fixed Point Lemma, we can find a Rosser sentence $R$ for $V$ such that $i$-EA $\vdash R \leftrightarrow \operatorname{prov}_{V}(\neg R) \leq \operatorname{prov}_{V}(R)$. We clearly have $i$-EA $\vdash \neg\left(R \wedge R^{\perp}\right)$. Using Rosser's argument, we can show that both $V+R$ and $V+\neg R$ are consistent. We note that both $R$ and $R^{\perp}$ can be rewritten over $i$-EA plus $\Sigma_{1}^{0}$-collection to the strict $\Sigma_{1}^{0}$ form. Since we work in extensions of $\mathrm{HA}$, we may assume that $R$ and $R^{\perp}$ are $\Sigma_{1}^{0}$.

We turn to the proof of theorem 4.6.

Proof Let $\Lambda$ and $U$ be as required for theorem 4.6. Consider any $\varphi$ such that $\Lambda \nvdash \varphi$. Let $\mathcal{K}$ be a counter-model in $\mathfrak{F}$. Suppose the non-endpoints of $\mathcal{K}$ are $a_{0}, \ldots, a_{n-1}$ and that we have chosen our enumeration in such a way that $a_{i} \preceq a_{j}$ implies $i \leq j$. We repeat our construction from the proof of theorem 4.1 of a sequence of models with $\mathcal{N}_{i}$ for $0, \ldots, n-1$, with the modification that we take as our base theory $W:=\mathrm{PA}+\operatorname{con}(U)$. So $\mathcal{N}_{n-1}$ will satisfy $\operatorname{con}(U)$ and incon $(\mathrm{PA}+\operatorname{con}(U))$.

We enumerate the endpoints as $b_{0}, \ldots, b_{r-1}$. We construct a sequence of $\Sigma_{1}^{0}$ sentences $S_{0}, \ldots, S_{r-1}$, with the following properties, for $i<j \leq r-1$ :

$$
W \vdash \operatorname{con}\left(U+S_{i}\right) \text { and } U \vdash \neg\left(S_{i} \wedge S_{j}\right) \text {. }
$$

The easiest way to construct such a sequence is by induction on $r$. If $r=1$, we take $S_{0}:=\top$. Suppose we have constructed $S_{0}^{\prime}, \ldots S_{s-1}^{\prime}$ with the desired properties. Let $R$ and $R^{\perp}$ be the pair consisting of the $\Sigma_{1}^{0}$ Rosser sentence for $U+S_{S-1}^{\prime}$ and its $\Sigma_{1}^{0}$ opposite. We take $S_{i}:=S_{i}^{\prime}$, for $i<r-1, S_{r-1}:=\left(S_{r-1}^{\prime} \wedge R\right)$, and $S_{r}:=\left(S_{r-1}^{\prime} \wedge R^{\perp}\right)$. We now construct in $\mathcal{N}_{n-1}$ inner Kripke models of $U+S_{i}$. The construction is analogous 
to the classical case. It is essential that the base theory in which we do the construction is classical! See Appendix A of [34] for a description of how to do an internal Kripke model construction. This gives us models $\mathcal{M}_{j}$ of $U+S_{j}$, internally definable in each $\mathcal{N}_{i}$.

We build a Kripke model for arithmetic by associating $\mathcal{N}_{i}$ with $a_{i}$ and $\mathcal{M}_{j}$ with $b_{j}$. This will be a model of $\mathrm{HA}+N$ by the internal version of Smoryński's Lemma 4.6, noting that the additional negations in $N$ are always downwards preserved. It will be a model of $\Lambda$ by the endpoint replacement property. We associate to each propositional atom $p$ in $\mathcal{K}$ the sentence:

$$
E_{p}:=\bigvee_{a_{i} \Vdash p}\left(C_{i} \wedge \operatorname{con}(U)\right) \vee \bigvee_{b_{j} \Vdash p} S_{j}
$$

As before in the proof of theorem 4.1, we transform $E_{p}$ to a classically equivalent $\Pi_{2}^{0}$-sentence, say $E_{p}^{+}$. Let $\sigma$ be the substitution $p \mapsto E_{p}^{+}$. By Ruitenburg's Lemma 4.3, we find that our new model does not force $\sigma(\varphi)$.

So, for example, for $\Lambda=$ IPC, $L C, K C, T_{n}$, we do have the de Jongh property for $\mathrm{HA}(\Lambda)$ plus the negation of the sentence expressing the Primitive Recursive Markov's Principle, and for $\mathrm{HA}(\Lambda)$ plus the negation of the sentence expressing the decidability of the Halting Problem. (See [27] for definitions of these properties.)

\section{A uniformization result}

In this section we show how to prove uniformization using recursion-theoretic arguments. This style of result is originally due to Franco Montagna [21] and, independently, Albert Visser [31].

Theorem 5.1 Consider any theory $T$ with elementary axiom set and any interpretation $N$, such that $N$ interprets $i$-EA, the constructive version of EA (which is also known as $\left.I \Delta_{0}+\exp \right)$, in $T$. Let $A_{i}$ be an elementary decidable sequence of sentences in the language of $T$.

Suppose, for every $i, T \nvdash A_{i}$. Then there is a $\Sigma_{1}^{0}$-formula $R(x)$ such that:

a. $i$-EA $\vdash(R(x) \wedge R(y)) \rightarrow x=y$,

b. for any $i, T \nvdash R^{N}(i) \rightarrow A_{i}$.

Proof Let $R(x)$ be such that:

$$
\begin{aligned}
i \text {-EA } \vdash R(x) \leftrightarrow & \exists p\left(\operatorname{proof}_{T}\left(p, R^{N}(x) \rightarrow A_{x}\right) \wedge\right. \\
& \left.\left.\forall q \leq p \forall y \leq q \neg \operatorname{proof}_{T}\left(q, R^{N}(y) \rightarrow A_{y}\right)\right)\right) .
\end{aligned}
$$

We work with the reasonable assumption that the code of the numeral of $y$ is larger than $y$, that the code of a formula in which a numeral occurs is larger than the code of that numeral, and that the code of a proof is larger than the code of any formula occurring in it. Also we assume that proofs have single conclusions. (See [12] for discussions of such reasonable assumptions.) 
We verify the uniqueness clause (a). Reason in $i$-EA. Suppose $R(x)$ and $R(y)$ with witnesses $p$ and $q$, respectively. In case $p=q$, we are done. Suppose $q<p$. From $R(x)$, we have: $\forall y^{\prime} \leq q \neg \operatorname{proof}_{T}\left(q, R^{N}\left(y^{\prime}\right) \rightarrow A_{y^{\prime}}\right)$. Since, by our assumptions on coding, we must have $y<q$, we find $\neg \operatorname{proof}_{T}\left(q, R^{N}(y) \rightarrow A_{y}\right)$. This contradicts $R(y)$.

We verify (b). Suppose, in order to derive a contradiction, that $T \vdash R^{N}(i) \rightarrow A_{i}$. Let $\pi$ be a proof that witnesses this fact. Via a finite search among proofs with Gödel number smaller or equal to the Gödel number of $\pi$, we may obtain the $T$-proof $\pi^{*}$ with smallest Gödel number with a conclusion of the form $R^{N}(j) \rightarrow A_{j}$. Say we have: $\pi^{*}: T \vdash R^{N}\left(i^{*}\right) \rightarrow A_{i^{*}}$. By the definition of $R$, it follows that $R\left(i^{*}\right)$ is true. So, by $\Sigma_{1}^{0}$-completeness, $T \vdash R^{N}\left(i^{*}\right)$. Hence, $T \vdash A_{i *}$, contradicting the assumption. We may conclude that $T_{i} \nvdash R(i) \rightarrow A_{i}$.

Let $\Lambda$ be any intermediate logic with the finite frame property. Suppose the set of finite frames $\mathfrak{F}$ corresponding to $\Lambda$ is decidable. We can easily find an elementary enumeration $\left(\varphi_{k}\right)_{k \in \omega}$ of all $\Lambda$-underivable formulas, such that we can find a countermodel $\mathcal{K}_{k}$ with frame in $\mathfrak{F}$ of $\varphi_{k}$ in an elementary way from $k$. In our main theorem, we have shown that we can transform $\mathcal{K}_{k}$ in an elementary way into a $\Pi_{2}^{0}$-substitution $\sigma_{k}$ such that $\mathrm{HA}(\Lambda) \nvdash \sigma_{k}\left(\varphi_{k}\right)$.

Theorem 5.2 Under the circumstances described above, we have the uniform de Jongh property for $\mathrm{HA}(\Lambda)$ with respect to $\Lambda$.

Proof Let $A_{i}:=\sigma_{i}\left(\varphi_{i}\right)$. Applying theorem 5.1, with $\mathrm{HA}(\Lambda)$ in the role of $T$, we find $R(x)$ with the promised properties. Take $\tau(p):=\exists y\left(R(y) \wedge \operatorname{true}_{\Pi_{2}^{0}}\left(\left\ulcorner\sigma_{y}(p)\right\urcorner\right)\right)$. Here true $_{\Pi_{2}^{0}}$ is the $\Pi_{2}^{0}$-truth predicate and $\left\ulcorner\sigma_{y}(p)\right\urcorner$ is an arithmetization that sends $y$ to the Gödel number of $\sigma_{y}(p)$.

Suppose $\mathrm{HA}(\Lambda) \vdash \tau\left(\varphi_{j}\right)$. It follows that $\mathrm{HA}(\Lambda) \vdash R(j) \rightarrow \sigma_{j}\left(\varphi_{j}\right)$, contradicting the assumption.

\section{Cautionary afterword}

We have proved our results with pleasant, pedestrian methods. The reason for this luxurious situation is the simplicity and power of the two basic ideas on which everything rests: Smoryński's idea of the preservation of HA under adding $\omega$ as a root and Smoryński's extension of the idea to non-standard models, provided that the rest of the Kripke model is definable in the new root. These ideas allow us to construct Kripke models step-by-step with a lot of control over their properties.

However, as Johan Cruijff said elk voordeel heb z'n nadeel, i.e., every advantage comes with a disadvantage. The disadvantage is that the essential dependence on these ideas makes the results not easily extendible.

A first possible direction of extension would be more frame classes. We note that there is not much hope to extend our results to frame classes with infinite models - at least not with the methods at hand. The reason is that the preservation of HA under adding a suitable root is essentially a tool for constructing finite objects. Of course, we may extend the construction to non-standardly finite models, but these are rather 
artificial from the point of view of frame classes of intermediate logics. The good news here is that almost all known natural logics do have the finite frame property. A possible exception is the Propositional Logic of Realizability. In this connection it is worth pointing out that de Jongh's original proof could handle infinite frames but that it did need trees.

The second possible extension would be more arithmetical theories. There seems to be no hope to extend our results to extensions of HA that lack the Smoryński property. For example, we do know that $\mathrm{HA}+\mathrm{ECT}_{0}$ has the de Jongh property of IPC, but the extension of $\mathrm{HA}+\mathrm{ECT}_{0}$ with intermediate logics is a complete terra incognita. In this case it could be interesting to look for counterexamples to the extension of our results.

Open Access This article is distributed under the terms of the Creative Commons Attribution Noncommercial License which permits any noncommercial use, distribution, and reproduction in any medium, provided the original author(s) and source are credited.

\section{References}

1. Blackburn, P., de Rijke, M., Venema, Y.: Modal Logic, Volume 53 of Cambridge Tracts in Theoretical Computer Science. Cambridge University Press, Cambridge, MA (2002)

2. Buss, S.: Intuitionistic validity in T-normal Kripke structures. Ann. Pure Appl. Log. 59, 159-173 (1993)

3. Ciardelli, I.A.: Inquisitive semantics and intermediate logics. Technical report, ILLC, University of Amsterdam, Amsterdam (2009)

4. Chagrov, A., Zakharyashev, M.: Modal Logic. Oxford Logic Guides. Oxford University Press, Oxford (1997)

5. de Jongh, D.H.J., Gabbay, D.: A sequence of decidable finitely axiomatizable intermediate logics with the disjunction property. J. Symbolic Log. 39, 67-78 (1974)

6. de Jongh, D.H.J., Visser, A.: Embeddings of heyting algebras. In: W. Hodges, M. Hyland, C. Steinhorn, J. Truss (eds) Logic at Work, Studies in Fuzziness and Soft Computing, pp. 187-213. Clarendon Press, Oxford (1996)

7. de Jongh, D.H.J.: The maximality of the intuitionistic predicate calculus with respect to Heyting's Arithmetic. J. Symbolic Log. 36, 606 (1970)

8. de Jongh, D.H.J., Smoryński, C.: Kripke models and the intuitionistic theory of species. Ann. Math. Log. 9, 157-186 (1976)

9. Friedman, H.: Some applications of Kleene's methods for intuitionistic systems. In: Mathias, A.R.D., Rogers, H. (eds.) Cambridge Summer School in Mathematical Logic, pp. 113-170. Springer Verlag, Berlin, Heidelberg, New York (1973)

10. Friedman, H.: Classically and intuitionistically provably recursive functions. In: Müller, G.H., Scott, D.S. (eds.) Higher Set Theory, pp. 21-27. Springer Verlag, Berlin, Heidelberg, New York (1978)

11. Gavrilenko, Yu.V.: Recursive realizability from the inuitionistic point of view. Sov. Math. Dokl. 23, 9-14 (1981)

12. Hájek, P., Pudlák, P.: Metamathematics of First-Order Arithmetic. Perspectives in Mathematical Logic. Springer, Berlin (1991)

13. Kleene, S.C.: Recursive predicates and quantifiers. Trans. AMS 53, 41-73 (1943)

14. Kleene, S.C.: Disjunction and existence under implication in elementary intuitionistic formalisms. J. Symbolic Log. 27, 11-18 (1962)

15. Kreisel, G., Putnam, H.: Eine Unableitbarkeitsbeweismethode für den Intuitionistischen Aussagenkalkül. Zeitschrift für Mathematische Logik and Grundlagen der Mathematik 3, 74-78 (1957)

16. Leivant, D.: Absoluteness in Intuitionistic Logic, volume 73. Mathematical Centre Tract, Amsterdam, 1979. The Thesis was originally published in 1975

17. Levin, V.A.: Some syntactic theorems on the calculus of finite problems of Yu.T. Medvedev. Sov. Math. Dokl. 10, 288-290 (1969) 
18. Maksimova, L., Shehtman, V., Skvortsov, D.: The impossibility of a finite axiomatization of Medvedev's logic of finitary problems. Sov. Math. Dokl. 20, 394-398 (1979)

19. McCarty, D.C.: Incompleteness in intuitionistic metamathematics. Notre Dame J. Formal Log. 32, 323-358 (1991)

20. Medvedev, Yu.: Interpretation of logical formulas by means of finite problems. Sov. Math. Dokl. 7, 180-183 (1966)

21. Montagna, F.: On the diagonalizable algebra of Peano Arithmetic. Boll. Unione Matematica Italiana 16-B, 795-813 (1979)

22. Plisko, V.E.: A survey of propositional realizability logic. Bull. Symbolic Log. 15, 1-42 (2009)

23. Rose, G.F.: Propositional calculus and realizability. Tran. Am. Math. Soc. 75, 1-19 (1953)

24. Solovay, R.M.: Provability interpretations of modal logic. Israel J. Math. 25, 287-304 (1976)

25. Smoryński, C.: Applications of Kripke models. In: Troelstra, A.S.(ed.) Metamathematical Investigations of Intuitionistic Arithmetic and Analysis, Springer Lecture Notes 344, pp. 324-391. Springer, Berlin (1973)

26. Tarski, A., Mostowski, A., Robinson, R.M.: Undecidable Theories. North-Holland, Amsterdam (1953)

27. Troelstra, A.S. (ed.): Metamathematical Investigations of Intuitionistic Arithmetic and Analysis. Springer Lecture Notes 344. Springer, Berlin (1973)

28. Troelstra, A.S., van Dalen, D.: Constructivism in Mathematics, vol. 2. Studies in Logic and the Foundations of Mathematics, vol. 123. North Holland, Amsterdam (1988)

29. van Oosten, J.: Exercises in Realizability. Ph. D. Thesis. Department of Mathematics and Computer Science, University of Amsterdam, Amsterdam (1991)

30. van Oosten, J.: A semantical proof of de Jongh's theorem. Arch. Math. Log. 31, 105-114 (1991)

31. Visser, A.: Aspects of Diagonalization and Provability. Ph.D. Thesis, Department of Philosophy, Utrecht University (1981)

32. Visser, A.: On the completeness principle. Ann. Mathe. Log. 22, 263-295 (1982)

33. Visser, A.: Evaluation, provably deductive equivalence in Heyting's Arithmetic of substitution instances of propositional formulas. Logic Group Preprint Series 4, Department of Philosophy, Utrecht University, Heidelberglaan 8, 3584 CS Utrecht, (1985)

34. Visser, A.: Interpretations over Heyting's Arithmetic. In: Orłowska E. (eds) Logic at Work, Studies in Fuzziness and Soft Computing, pp. 255-284. Physica-Verlag, Heidelberg/New York (1998)

35. Visser, A.: Rules and arithmetics. Notre Dame J. Formal Log. 40(1), 116-140 (1999)

36. Visser, A.: Substitutions of $\Sigma_{1}^{0}$-sentences: explorations between intuitionistic propositional logic and intuitionistic arithmetic. Ann. Pure Appl. Log. 114, 227-271 (2002)

37. Visser, A.: Can we make the Second Incompleteness Theorem coordinate free? J. Log. Comput. (2009). doi:10.1093/logcom/exp048

38. Yang, F.: Intuitionistic subframe formulas, NNIL-formulas and n-universal models. Technical report, ILLC, University of Amsterdam, Amsterdam (2008) 